\title{
INTELLIGENT HYDRAULIC POWER GENERATING GROUP
}

\author{
Mihai Avram¹, Valerian-Emanuel Sârbu², Alina-Rodica Spânu ${ }^{3}$, Constantin Bucșan ${ }^{4}$ \\ 1,2,3,4 POLITEHNICA University of Bucharest, 313 Spl. Independentei, Bucharest, Romania \\ E-mail: mavram02@yahoo.com
}

\begin{abstract}
The domain of hydraulics is expensive, but this is changing due to the new manufacturing technologies. The hydraulic energy generators without an intelligent controller have low efficiency and they will be replaced by intelligent ones. The use of an inverter can significantly reduce the energy consumption by modifying the speed of the motor in order to obtain the flow/pressure required by the application. If a variable flow pump is used in the system, the energy saving is important, due to the pump working with the optimum speed, pressure and flow needed for a maximum efficiency. The use of systems connected to the Internet (and cloud services) increases the reliability due to the predictive maintenance and the reduced working costs. The paper is the starting point in developing an intelligent control system using optimized algorithms for decreasing the consumption, increasing the reliability, reducing the noise and increasing the operating safety.
\end{abstract}

Keywords: Hydraulic Power Supply, Intelligent System, Actuator.

\section{Introduction}

Nowadays it is about "the fourth industrial revolution” or simply „Industry 4.0”. The key of this new stage is represented by the interconnected digital systems. The manufacturing processes will become self-organized processes where human operators, machines, devices, logistics and products communicate and cooperate directly with one another. This interconnecting network must cover all the stages of the product development: concept, design, manufacture, installation, service, till recycling. In such an "intelligent factory" the products pass independently through the manufacturing processes and are easily identified and localized at any moment, giving flexibility and individualization to mass production.

Cyber-Physical Systems are complex systems capable to control and coordinate physical and organizational processes at local and global level using information and communication technology.

They are the basis of control architectures for complex systems, containing autonomous subsystems as physical processes, sensors networks, communicating systems or software services. The global behaviour of such a system is given by the interaction of the components. Here are some examples of such systems: transportation systems, energetic systems, factories systems, intelligent manufacturing systems, smart-cities etc.

Two basic components are required in order to create such systems: intelligent equipment and specialists capable to develop, implement and operate the systems.
Nowadays main trends are digitization, internet of things, internet of services and cyber physical systems. Mechatronics will have an essential contribution in this domain.

A mechatronic product is characterized by:

- multifunction - the capability to accomplish different tasks with one hardware structure by modifying the working program;

- intelligence - the capability to communicate with the environment and to make decisions when disturbances appear;

- flexibility - the possibility to easily modify the structure of the system in the stagers of design, manufacture or operation, due to the modular structure of the system;

- the possibility to be remote controlled and to communicate with other intelligent systems; this require complex communication interfaces;

- permanent technical evolvement, in order to meet the requirements of the changing market using the new manufacturing technologies (CNC, robots, miniaturization, nano-technologies etc.).

A system is considered to be intelligent when it has the capability to work correctly for a long period of time in uncomplete defined environments without external intervention. This requires a hardware structure that allows the system to have intelligent reactions to the disturbances: the control unit (using a microprocessor, a microcontroller, a programmable automaton or a PC) and a network of sensors providing information on the state of the mechanical structure and on the working environment. 
The control unit analyses the information coming from the operator and from the sensors, evaluates the state of the system and of the working environment and generates control signals to the effectors in order to carry out the programmed task. In exceptional cases, such as interferences or conflict of interests, signals are transmitted to the upper level.

This in one of the four basic principles of Industry 4.0 [1].

These systems have communication interfaces and can be connected in a network with machines, devices, sensors and people, so meeting another basic principle of Industry 4.0.

\section{Hydronic Systems}

The development of proportional valves marked a qualitative leap in the domain of hydraulic actuating systems. Hydraulic proportional devices for controlling hydraulic power were developed using performant electro-mechanical actuators and new types of sensors. Using such devices in the structure of the hydraulic actuating systems led to the informatization of the systems and to the development of a new subdomain of mechatronics: hydronics - a synergetic joining of hydraulics, electronics and informatics.

A hydronic system also contains classic hydraulic devices, sensors and transducers, electronic circuits for data processing, A/D and D/A convertors, controllers or microprocessors. Using advanced control theories, these systems can control pressure and flow rate, displacement, speed and acceleration, and also forces and torques developed by the actuators of the system. Such systems are used when automated control of the working stages is required, when speed levels shift is needed for high accuracy positioning, and when ease and flexibility of programming is required.

The designers of hydraulic control devices try to adept the other components of the system (motors, power supply groups) in order to be easily integrated in complex hydronic systems. So, a new generation of "intelligent devices" is born. This paper deals with such a system.

\section{Hydraulic Power Supply Groups}

The hydraulic power supply group is used to supply the flow rate of oil to the actuating system for a certain maximum working pressure. It also must maintain the temperature of the fluid within the normal limits and must assure its required purity. Usually, such a group contains the following devices: pump, tank, heat exchanger, filters etc.:
- the tank provides the fluid supply needed for the system working, protects the fluid against elements and polluting, allows the decantation of the transported particles and the separation of water and air and also contributes to the cooling of the fluid;

- the pump generates the hydraulic energy required by the system;

- the filters maintain the purity of the fluid;

- the heat exchanger maintains the temperature of the fluid under a certain limit.

The pump can be with fix or adjustable flow rate.

The flow supplied by the pump is given by:

$q=\frac{V_{P} \cdot n}{1000} \cdot \eta_{v}[l / \mathrm{min}]$

where:

$V_{P}$ is the cylinder capacity of the pump $\left[\mathrm{cm}^{3}\right]$;

$n$ is the rotation speed of the actuating shaft $[\mathrm{rot} / \mathrm{min}]$;

$\eta_{v}$ is the volumetric efficiency of the pump.

Usually the actuating motor (electrical or thermal) of the pump has a constant rotation speed.

The pumps used in industrial hydraulic systems are actuated by asynchronous electric motors, which are robust and cheap. In such a case, the variation of the flow rate is obtained by modifying the cylinder capacity of the pump. Adjusting and stabilizing devices are commonly used: the adjusting devices require the intervention of an operator or a control signal (electric, hydraulic or pneumatic), while the stabilizing devices automatically adjust the cylinder capacity according to the flow rate, the pressure or the power according to a certain low [3].

Another method of increasing importance is the actuation of the pump with an electric motor with variable rotation speed.

The variation of the rotation speed $\mathrm{n}$ in the domain $\left[0, n_{n}\right]$ determines the variation of the flow rate $q$ :

$q=q_{n} \frac{n}{n_{n}}$

The pressure drop on the output orifice of the pump, $\Delta p$, is given by:

$\Delta p=\frac{1}{s_{n}} \cdot \frac{\rho}{2} \cdot q^{2}=k \cdot q^{2}$

or as a function of $\frac{n}{n_{n}}$ :

$\Delta p=\Delta p_{n} \cdot\left(\frac{n}{n_{n}}\right)^{2}$

The hydraulic power supplied by the pump is given by:

$P_{h}=p \cdot q$

or as a function of $\frac{n}{n_{n}}$ :

$P_{h}=P_{h n} \cdot\left(\frac{n}{n_{n}}\right)^{3}$

Equations (1), (4) and (6), in a dimensionless form, are represented in Fig. 1. 


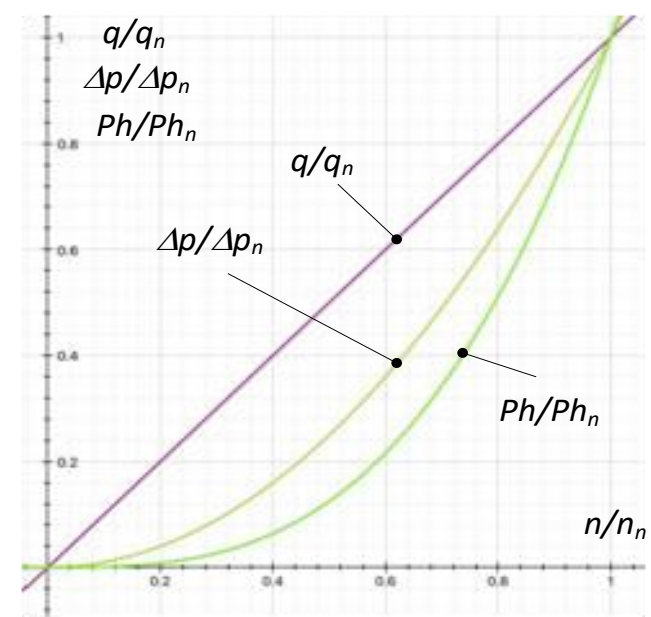

Figure 1: Graphicrepresentation of eq. (1), (4) and (6)

So, the hydraulic power for a hydraulic actuating system can be obtained using a pump of the following type [2]:

1. with fixed cylinder capacity, actuated by a constant speed motor;

2. with adjustable cylinder capacity, actuated by a constant speed motor;

3. with fixed cylinder capacity, actuated by a variable speed motor;

4. with adjustable cylinder capacity, actuated by a variable speed motor.

The type of the hydraulic power generator must be chosen after a techno-economic analysis. The cost for the life cycle of a technical system has the following structure:

- $\quad 5 . .20 \%$ - investing costs;

- $\quad 80 . . .95 \%$ - operating costs: installation, training, spare parts, maintenance costs, employment costs, taxes, assurances, energy, modernization etc.

In the case of hydraulic actuating systems, where very high power is required, the costs with the energy are preponderant.

The following structures of a simple hydraulic actuating system can be used:

- Actuating system with a fixed flow pump - Fig. 2, with the following characteristics:

- low acquisition cost;

- low energetic efficiency; the most significant power loss $\Delta N_{E R C}$ is located at the level of the adjusting and control devices $E R C$

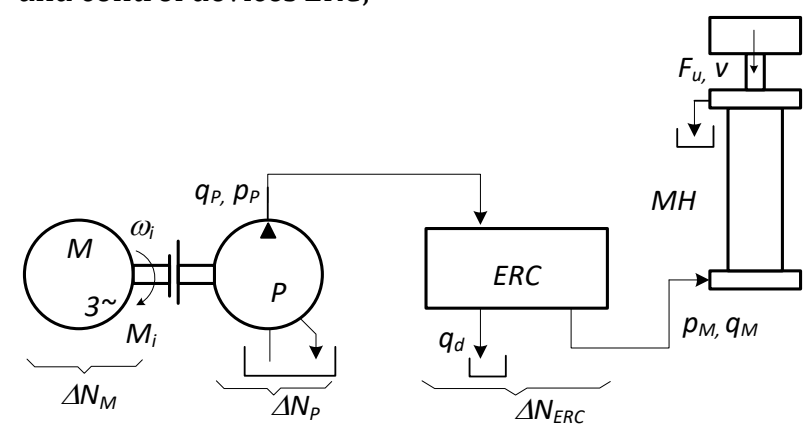

Figure2: Actuating system with a fixed flow pump
- the electric motor $M$ with constant speed is coupled all the time, even when the system is not loaded, and its shaft is rotating with constant speed;

- $\quad$ high power loss $\Delta N_{P}$, at the level of the pump $P$, as a consequence of the high actuating rotation speed;

- $\quad$ low efficiency of the motor and pump when the hydraulic actuator is partially loaded.

- Actuating systems with adjustable cylindric capacity pump - Fig. 3, with the following characteristics:
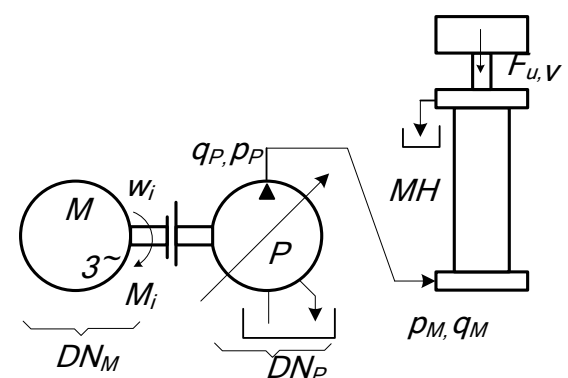

Figure 3: Actuating systems with adjustable cylindric capacity pump

- $\quad$ high acquisition cost, due to the complexity of the pump;

- $\quad$ can be provided control devices;

- the control devices block can be eliminated or simplified, so the power loss at this level can be significantly reduced;

- $\quad$ the flow rate and the pressure at the output of the pump assures the speed and the torque needed at the shaft of the actuator.

- Actuating system with adjustable cylindric capacity pump actuated by a variable speed motor Fig. 4, with the following characteristics:

- the power loss $\Delta N_{E R C}$ was eliminated;
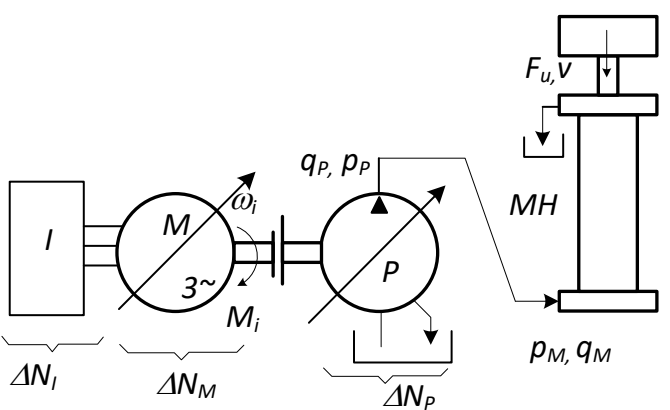

Figure 4: Actuating system with adjustable cylindric capacity pump actuated by a variable speed motor

- the rotation speed is reduced with $40 \ldots 50 \%$ when the load is partial;

- the efficiency of the pump and the motor increases when the load of the actuator is partial;

- $\quad$ the power loss at the level of the pump are reduced due to the lower actuating speed;

- $\quad$ a supplementary power loss appears at the level of the invertor. 
Nowadays, when the cost of energy is high, many manufacturers offer pumps with fixed cylindric capacity and a system for variating the speed of the actuating motor. An example is the firm Parker, who developed a pump with Denisson paddles, which reduces the energetic consumption by $50 \%$.

Another example is the firm Bosch Rexroth, with the Sytronix range [4].

\section{The Proposed Intelligent Hydraulic Group}

The proposed system is based on a hydraulic power generating group (Fig. 5, the blue rectangle), consisting of the pump $P$ with fixed cylindric capacity, actuated by an asynchronous motor $M$., the tank $R z$ and the safety valve $S p$.

This structure was transformed in an intelligent one as shown in Fig. 5, and has the following characteristics:

- the supplied flow $q_{R P}$ is according to the system needs;

- the fluid pressure $p_{R P}$ is able to actuate the maximum load of the system;

- the fluid temperature is maintained in the regime range $t_{r}=60 \ldots 65^{\circ} \mathrm{C}$;

- the purity of the fluid meets the requirements of the actuated system;

- the level of the fluid in the tank can be monitored and a value under the lower limit is signalled.

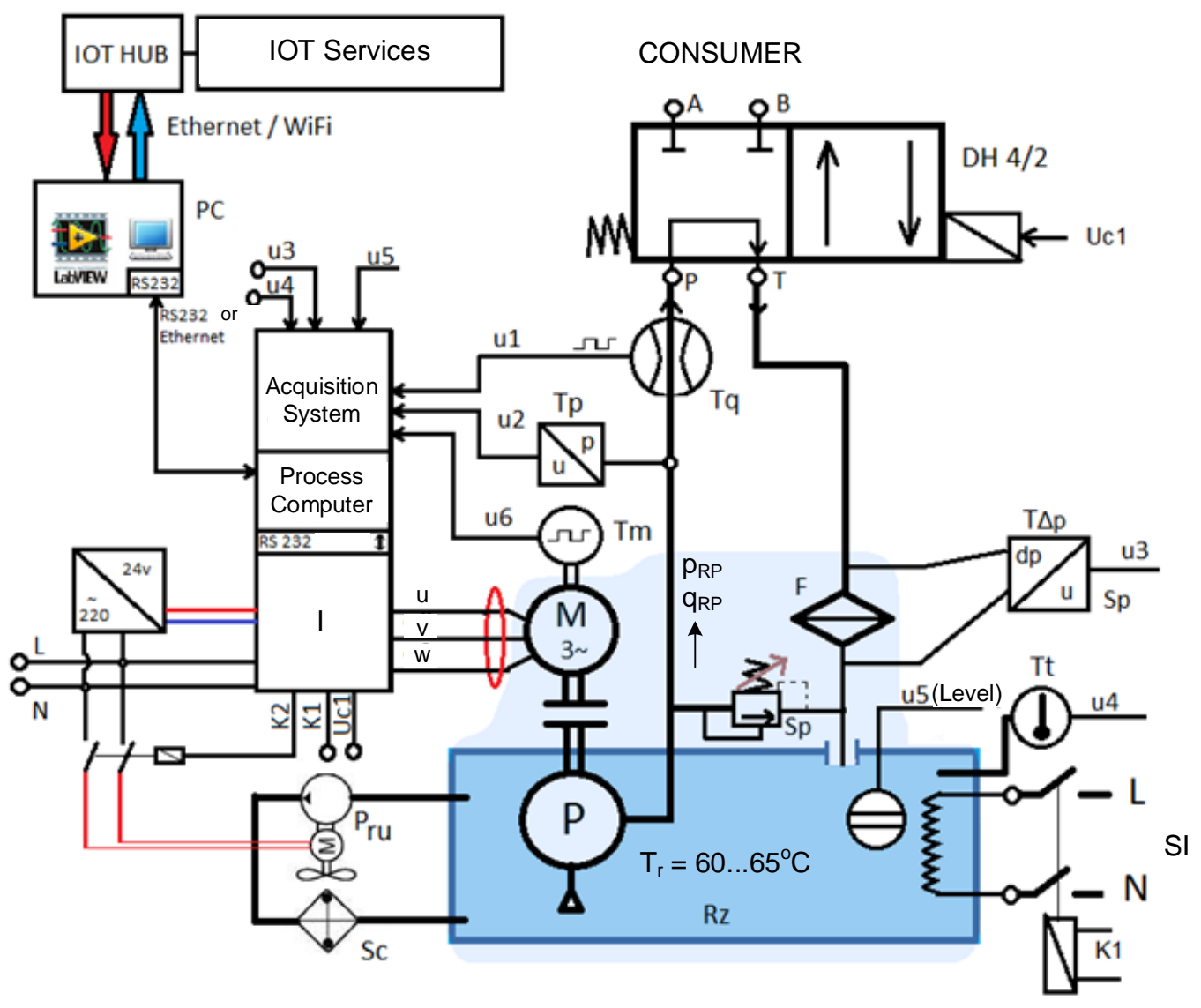

Figure 5: The proposed intelligent hydraulic group

The group can communicate with the control unit of the hydronic system in which was integrated.

The presented characteristics are achieved due to the following devices:

- a three-phased invertor - it controls the speed of the asynchronous motor and so the flow $q_{R P}$ supplied by the pump;

- an oil heating system $S I$ - it can be coupled or uncoupled by a relay $K_{1}$; this device is useful when starting the system, until the temperature of the fluid reaches the regime value;

- an oil cooling system $S R$ - it can be coupled or uncoupled by a relay $K_{2}$; this system consists of a fixed flow pump $P_{r u}$ and a heat exchanger $S C$ and it is coupled when the oil temperature exceeds the maximum limit of the regime range;

- transducers for fluid parameters: pressure $T_{p}$, pressure difference - $T_{P}$, flow - $T_{q}$, temperature $T_{t}$, level - $T_{n i}$, rotation speed - $T_{n}$;

- a $4 / 2$ hydraulic valve with preferential position and electrical command.

The Inverter I (Fig. 6) was designed and built with commercial electronic components, in order to lower the costs. 


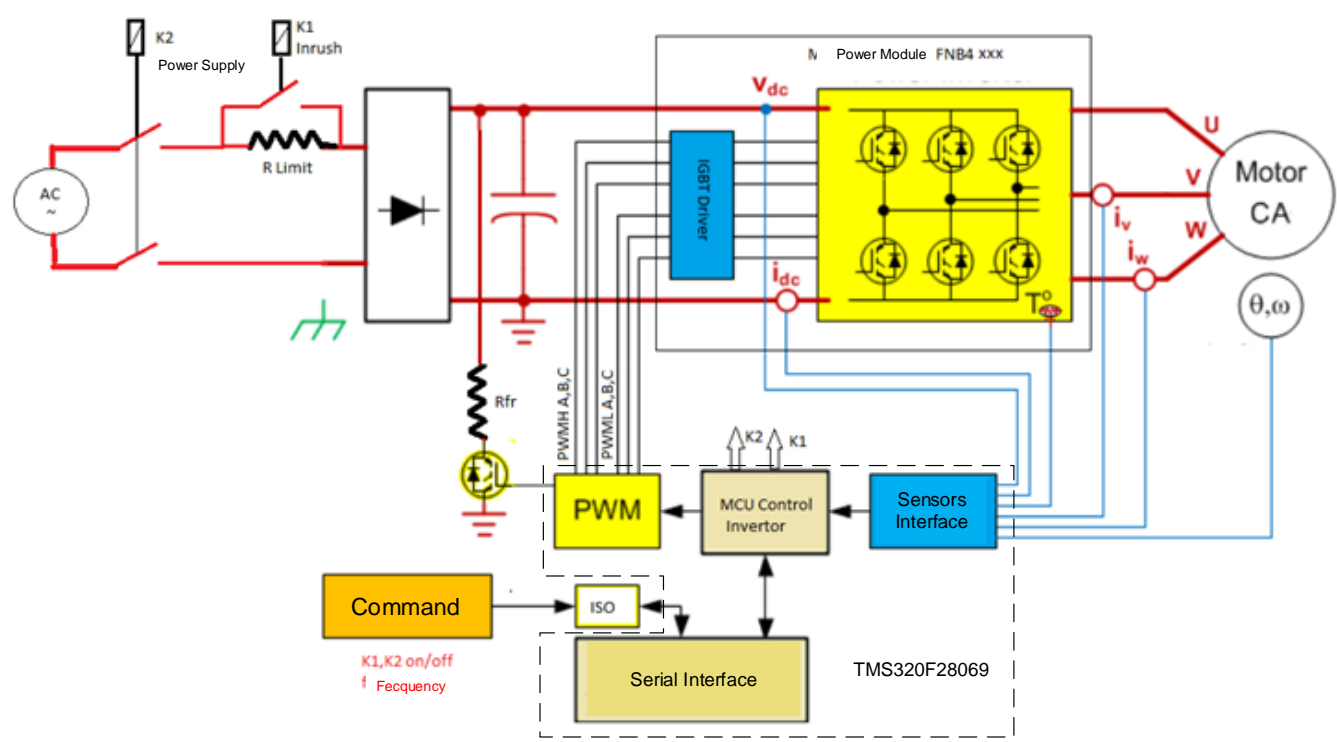

Figure 6: The three-phased invertor

The basic component of the inverter [6] is the hybrid integrated circuit FNB43060T2 - it contains the high-power transistors and the circuits needed to activate their gates [7].

The microcontroller TMS320F28069 [8] from Texas Instruments was used to control the power transistors using 3 PWM channels with complementary output.

In order to generate the PWM signals, the method of comparing the signal to be modulated with a sawtooth signal with a frequency of $16 \mathrm{kHz}$ (or $5 \mathrm{kHz}$ ) was used. The modulated signal is a sinusoidal one, with the amplitude calculated as a function of the frequency $f_{e}$ needed at the output of the inverter, respecting the condition that the ratio voltagefrequency is constant.

The modulated generated pulses are of low voltage, $[0,3] \mathrm{V}$, and they cannot be used to control a motor. The power module FNB43060T2 has the role to transform the PWM pulses of low voltage in PWM pulses of high voltage.

The amplitude of the fundamental harmonic [5] corresponding to the line voltage generated by the inverter depend on the dc supply voltage $U_{d}$ and it can be proportionally adjusted using the amplitude modulation index $m_{a}$ :

$$
U_{l}=0,612 \cdot m_{a} \cdot U_{d}
$$

The effective value of the fundamental harmonic of the phase voltage is given by:

$$
U_{f}=0,353 \cdot m_{a} \cdot U_{d}
$$

In Romania, the line voltage is $400 \mathrm{~V}$. The DC voltage $U_{d}$ is obtained using a diode rectifier and is given by:

$U_{d}=\sqrt{2} \cdot 400=565 \mathrm{~V}$.

These equations can be used to calculate the effective values of the line and phase voltage at the output of the inverter, for $m_{a}=1: U_{l} \approx 345 \mathrm{~V}$ and
$U_{f} \approx 200 \mathrm{~V}$. These values are not sufficient to supply an AC motor in nominal conditions.

An increase of $15 \%$ of the supply voltage is obtained by injecting a third order harmonic in the sinusoidal signal [6],[11].

The proposed system is provided with a process computer - it receives the data from the transducers within the system and uses a PI/PID algorithm to generate the required command. The computer communicates with the MCU Control Inverter using an isolated serial interface at 115.2 kbaud. The recognized commands are the following:

- set the inverter frequency;

- activate the safety switch;

- activate the capacitors relay;

- read the inverter parameters (current, voltage, temperature);

- activate the brake.

Using the algorithms developed by the authors, working programmes were written and stored in the process computer memory.

The main algorithms are:

- start/stop the pump;

- automated working regime;

- safety system stop.

The process computer also assures the protection of the supplied system in the following situations:

- the motor or the pump is blocked;

- the temperature exceeds the superior limit of the regime range - the power supply generating group is deactivated;

- the working fluid quality does not correspond to the requirements - the $4 / 2$ hydraulic valve is uncoupled;

- the oil level in the tank is not in the regime range the power supply generating group is deactivated. 
The process computer can be controlled or monitored by an upper level system. In order to connect to the internet of things, an intermediate for the protocol conversion is needed. LabView programming environment contains libraries using the MQTT protocol for communication with aIoT hub, that redirects the messages to long term storage solutions, events processing systems or other devices as phones or tablets.

\section{Conclusions}

The domain of hydraulics is expensive, but this is changing due to the new manufacturing technologies. The hydraulic energy generators without an intelligent controller have low efficiency and they will be replaced by intelligent ones. The control systems can be built using commercial components, leading to important energy savings only by controlling the process and managing the excess generated energy.

The use of an inverter can significantly reduce the energy consumption by modifying the speed of the motor in order to obtain the flow/pressure required by the application. Some modifications are needed (the cooling of the motor is not sufficient at low speed, the shaft must be grounded to avoid electric discharges).

If a variable flow pump is used in the system, the energy saving is important, due to the pump working with the optimum speed, pressure and flow needed for a maximum efficiency.

The use of systems that are connected to the Internet (and cloud services) increases the reliability due to the predictive maintenance and the reduced working costs. Implementing the IoT (Industry 4.0) is simple in the case of applications developed on normal computers, especially when using the graphic programming environment LabView.

The paper is the starting point in developing an intelligent control system using optimized algorithms for decreasing the consumption, increasing the reliability, reducing the noise and increasing the operating safety.

\section{References}

[1] Mario Hermann, Tobias Pentek, Boris Otto: Design Principles for Industrie 4.0 Scenarios, 2016 49th Hawaii International Conference on System Sciences (HICSS), 10.1109/HICSS.2016.488;

[2] http://www.debizz.ro/bosch-utilizeazaindustria-4-0-pentru-a-si-crestecompetitivitatea/

[3] http://www.hydraulicspneumatics.com/sites/hy draulicspneumatics.com/ files/uploads/2012/10/Komsta_Rexroth_9_11.pd $\mathrm{f}$;

[4] https://www.boschrexroth.com/ics/cat/?id=\&ca $\mathrm{t}=$ Industrial-Hydraulics-Catalog\&p=g284382;

[5] Albu Mihai: Electronică de putere, Casa de Editură Venus;

[6] AC Induction Motor Control Using Constant V/Hz Principle and Space Vector PWM,http://www.ti.com/lit/an/spra284a/spra2 84a.pdf;

[7] FNB41560: Intelligent Power Module,http://www.onsemi.com/PowerSolution s/product.do?id=FNB41560;

[8] TMS320F28069 (ACTIVE) Piccolo Microcontroller,http://www.ti.com/product/TM S320F28069;

[9] Scalar (V/f) Control of 3-Phase Induction Motors,http://www.ti.com/lit/an/sprabq8/spra bq8.pdf;

[10] PROJECT \#2 SPACE VECTOR PWM INVERTER,http://www2.ece.ohiostate.edu/ems/PowerConverter/SpaceVector_P WM_Inverter.pdf;

[11] A Practical Guide for Connecting LabVIEW to the Industrial IoT,http://www.ni.com/whitepaper/53954/en/. 